\title{
Asymptotic estimates for some number-theoretic power series
}

by

\author{
Stefan Gerhold (Vienna and Orsay)
}

$$
\begin{array}{r}
\text { Such functions as } \sum \mu(n) x^{n}, \\
\sum \phi(n) x^{n}, \sum \Lambda(n) x^{n} \text { are extremely difficult to handle. } \\
\text { - G. H. Hardy, E. M. Wright [15] }
\end{array}
$$

1. Introduction. While Hardy and Wright are of course right in that ordinary generating functions of arithmetic functions do not share the versatility and usefulness of their well-known Dirichlet counterparts, several non-trivial results - both old and new-have been obtained for them. For instance, the analysis of

$$
\sum_{n=1}^{\infty} \tau(n) z^{n}=\sum_{n=1}^{\infty} \frac{z^{n}}{1-z^{n}}
$$

where $\tau(n)$ denotes the number of divisors of $n$, goes back to Lambert [16], and the expansion

$$
\begin{aligned}
\sum_{n=1}^{\infty} \tau(n) e^{-n t} \sim \frac{1}{t} \log \frac{1}{t}+\frac{\gamma}{t}-\sum_{n=0}^{\infty} \frac{B_{n+1}^{2}}{(n+1) !(n+1)} t^{n}, \\
\text { where } t \rightarrow 0,|\arg (t)|<\frac{1}{2} \pi-\theta \text { for some } \theta>0,
\end{aligned}
$$

involving Euler's constant and Bernoulli numbers, has been known for a long time [13, 18, 22, 24]. Titchmarsh [22] has applied (1) in a result on mean values of the Riemann zeta-function, and Canfield et al. 9. have extended (1) to the case of the arithmetic function that counts only divisors in some fixed residue class. Another generalization has been obtained by Berndt and Evans 6], who also proved the formula

$$
\sum_{n=1}^{\infty} p_{n} z^{n} \sim \frac{1}{(1-z)^{2}} \log \frac{1}{1-z}, \quad z \rightarrow 1^{-} \text {in } \mathbb{R},
$$

where $p_{n}$ is the $n$th prime number.

2010 Mathematics Subject Classification: Primary 11N37; Secondary 30B10.

Key words and phrases: arithmetic function, asymptotics, power series, Mellin transform. 
Recently, the transcendence of number-theoretic power series has been of interest to several authors. Banks et al. 22 have established the irrationality of $\sum \mu(n) z^{n}, \sum p_{n} z^{n}, \sum \tau(n) z^{n}$, and several other similar series, over $\mathbb{Q}(z)$. Later it was noted [4, 8] that the transcendence of these series follows easily from the fact that they have the unit circle as a natural boundary. This property even shows that they are not $D$-finite [5, 12, 20. General results about the transcendence of $\sum f(n) z^{n}$ with $f$ multiplicative have recently been obtained by Borwein and Coons [8] and by Bell and Coons [4].

The present note is concerned with asymptotic estimates for power series $\sum a_{n} z^{n}$, where the Dirichlet generating function $\sum a_{n} n^{-s}$ has singularities at the zeros of the Riemann zeta-function. For instance, $a_{n}=\mu(n)$ falls under this category. Delange [11] has noted that the prime number theorem in the form

$$
M(x):=\sum_{n \leq x} \mu(n)=\mathrm{o}(x), \quad x \rightarrow \infty,
$$

where $M(x)$ denotes the Mertens function, readily implies

$$
\sum_{n=1}^{\infty} \mu(n) z^{n}=\mathrm{o}\left(\frac{1}{1-z}\right), \quad z \rightarrow 1^{-} \text {in } \mathbb{R} .
$$

A quick way to improve this starts from Walfisz' deep result [23]

$$
M(x)=\mathrm{O}\left(x \exp \left(-\frac{c(\log x)^{3 / 5}}{(\log \log x)^{1 / 5}}\right)\right)
$$

for some $c>0$. Recall the following basic Abelian theorem [7, 12, 19]:

LEMmA 1. Suppose that $\left(a_{n}\right)$ is an ultimately monotone real sequence with $a_{n} \sim n^{\alpha} \ell(n)$, where $\alpha>0$, and $\ell$ is positive and varies slowly at infinity. Then

$$
\sum_{n=1}^{\infty} a_{n} z^{n} \sim \frac{\Gamma(\alpha+1)}{(1-z)^{\alpha+1}} \ell\left(\frac{1}{1-z}\right)
$$

as $z \rightarrow 1$ in any sector

$$
S_{\theta}:=\left\{z \in \mathbb{C}:|\arg (1-z)| \leq \frac{1}{2} \pi-\theta\right\}, \quad \theta>0 .
$$

From the lemma (applied here only for real $z$ ) and (2) we obtain

$$
\sum_{n=1}^{\infty} \mu(n) z^{n}=(1-z) \sum_{n=1}^{\infty} M(n) z^{n}=\mathrm{O}\left(\frac{1}{t} \exp \left(-\frac{c(\log 1 / t)^{3 / 5}}{(\log \log 1 / t)^{1 / 5}}\right)\right)
$$

where

$$
t=-\log z \sim 1-z \rightarrow 0^{+} \quad \text { in } \mathbb{R} .
$$

There seems to be no Tauberian result available to translate (4) back into an estimate for the Mertens function $M(x)$. This typical asymmetry suggests that we might be able to do a little better than (4) by using dedicated 
methods. Indeed, our main result (Theorem 4 below) yields the following improvement.

THEOREM 2. If $z$ tends to 1 in an arbitrary sector of the form (3), then

$$
\begin{aligned}
\sum_{n=1}^{\infty} \mu(n) z^{n}=\mathrm{O}\left(\frac{1}{t} \exp \left(-\frac{0.0203 \log 1 / t}{(\log \log 1 / t)^{2 / 3}(\log \log \log 1 / t)^{1 / 3}}\right)\right) & \\
t & =-\log z .
\end{aligned}
$$

The proof rests on the contour integral representation [22]

$$
\sum_{n=1}^{\infty} \mu(n) e^{-n t}=\frac{1}{2 \pi i} \int_{\kappa-i \infty}^{\kappa+i \infty} \frac{\Gamma(s)}{\zeta(s)} t^{-s} d s, \quad \kappa>1 .
$$

In a way that is familiar from the prime number theorem or the SelbergDelange method [21], one can deform the integration contour a little bit into the critical strip $0<\Re(s)<1$, and then estimate the resulting integral. The exponential decrease of the Gamma function along vertical lines is a convenient feature of (6), which is not present in the Perron summation formula [21]

$$
\sum_{n \leq x} \mu(n)=\frac{1}{2 \pi i} \int_{\kappa-i \infty}^{\kappa+i \infty} \frac{1}{\zeta(s)} \frac{x^{s}}{s} d s, \quad \kappa>1, x \in \mathbb{R}^{+} \backslash \mathbb{Z} .
$$

Power series thus tend to be easier to estimate than summatory functions. The fact that $x$ is real in (7), whereas in (6) it is natural to consider also complex $t$, causes no great difficulties (at least if $|\arg (t)|$ stays bounded away from $\frac{1}{2} \pi$ ).

In the following section we put (5) into perspective by relating the growth of $\sum \mu(n) z^{n}$ to the Riemann Hypothesis. Section 3 contains our main result, from which (5) follows. A few related power series are estimated in Section 4 . Section 5 collects some open problems.

2. Connection to the Riemann Hypothesis. In conjunction with (2) and (5), the following proposition shows that the gap between the Riemann Hypothesis and what is provable today is slightly smaller in the power series case than in the case of the summatory function $M(x)$.

Proposition 3. Let $1 / 2 \leq \eta<1$. Then the following are equivalent:

(i) $\zeta(s)$ has no zeros for $\Re(s)>\eta$,

(ii) $M(x)=\mathrm{O}\left(x^{\eta+\varepsilon}\right)$,

(iii) $\sum_{n \geq 1} \mu(n) z^{n}=\mathrm{O}\left((1-z)^{-\eta+\varepsilon}\right)$ as $z \rightarrow 1^{-}$in $\mathbb{R}$.

Proof. The equivalence of (i) and (ii) is classical for $\eta=\frac{1}{2}$ (see Titchmarsh [22]), and the proof of the more general case is an easy modification. 
(The implication (ii) $\Rightarrow(\mathrm{i})$, which we actually do not require, is posed as Exercise 13.4 in Apostol's textbook [1.) If (ii) holds, then (iii) follows by Lemma 1. Finally, if we assume that (iii) is true, we have

$$
F(t):=\sum_{n=1}^{\infty} \mu(n) e^{-n t}=\mathrm{O}\left(t^{-(\eta+\varepsilon)}\right), \quad t \rightarrow 0^{+} \text {in } \mathbb{R} .
$$

Hence the Mellin transform [13]

$$
\int_{0}^{\infty} F(t) t^{s-1} d t=\frac{\Gamma(s)}{\zeta(s)}
$$

defines an analytic function for $\Re(s)>\eta$.

Under the Riemann Hypothesis, one would expect that we can push the integration contour in (6) across the critical line $\Re(s)=\frac{1}{2}$ to obtain an expansion of the form

$$
\sum_{n=1}^{\infty} \mu(n) e^{-n t} \stackrel{?}{=} t^{-1 / 2} H(\log 1 / t)-2+\mathrm{o}(1), \quad t \rightarrow 0,
$$

where $H$, a bounded oscillating function, is a sum of infinitely many harmonics corresponding to the non-trivial zeros of the zeta-function. The fast decrease of the Gamma function makes the residues of $\Gamma(s) / \zeta(s)$ at these zeros rather small, so that the term -2 will dominate in (8) unless $1-z$ is very close to zero. Indeed, the $\Omega\left(t^{-1 / 2}\right)$ term becomes numerically visible only from about $1-z=10^{-10}$ onwards [P. Flajolet, private communication]. This "fake asymptotics" property has also been noted by Bateman and Diamond [3. Without assuming the Riemann Hypothesis, Delange [11] has shown that

$$
\sum_{n=1}^{\infty} \mu(n) z^{n}=\Omega_{ \pm}\left(\frac{1}{\sqrt{1-z}}\right), \quad z \rightarrow 1^{-} \text {in } \mathbb{R},
$$

which is in line with (8), and shows that the left-hand side of (8) does not converge to -2 .

3. Main result. We write

$$
D(s)=\sum_{n=1}^{\infty} \frac{a_{n}}{n^{s}}, \quad s=\sigma+i \tau,
$$

for the Dirichlet generating function of a sequence $\left(a_{n}\right)$. The following theorem gives an estimate for the power series $\sum a_{n} z^{n}$ near $z=1$, assuming analyticity and growth conditions for $D(s)$.

THEOREM 4. Let $\left(a_{n}\right)$ be a sequence of complex numbers such that $D(s)$ is absolutely convergent for $\Re(s)>1$ and has an analytic continuation to a 
set $\Omega$ of the form

$$
\sigma \geq g(\tau):= \begin{cases}1-b(\log |\tau|)^{-\alpha}(\log \log |\tau|)^{-\beta}, & |\tau| \geq w \\ 1-b(\log w)^{-\alpha}(\log \log w)^{-\beta}, & |\tau| \leq w\end{cases}
$$

for some positive parameters $\alpha, \beta, b, w$. Assume furthermore that

$$
D(s)=\mathrm{O}\left(\tau^{\nu}\right)
$$

uniformly as $s \rightarrow \infty$ in $\Omega$, for some $\nu>0$. Then for any $\varepsilon>0$,

$$
\begin{aligned}
\sum_{n=1}^{\infty} a_{n} z^{n}=\mathrm{O}\left(\frac{1}{t} \exp \left(-\frac{(b-\varepsilon) \log 1 / t}{(\log \log 1 / t)^{\alpha}(\log \log \log 1 / t)^{\beta}}\right)\right) & \\
t & =-\log z \sim 1-z .
\end{aligned}
$$

The variable $z$ may tend to 1 in an arbitrary sector of the form (3).

This result immediately implies the bound (5), by noting that $D(s)=$ $1 / \zeta(s)$ for $a_{n}=\mu(n)$ and putting $\alpha=\frac{2}{3}$ and $\beta=\frac{1}{3}$. The required analyticity and growth of $1 / \zeta(s)$ are the content of Korobov and Vinogradov's famous theorem [22], which describes the largest known zero-free region for the Riemann zeta-function. (Recall that it leads to the best known error term in the prime number theorem.) For the constant $b$ in $(9)$ one may take $b=$ $0.05507 \times(4.45)^{-2 / 3}>0.0203$ in this case, by a result of Ford [14].

Proof of Theorem 4. The convergence assumption on $D(s)$ clearly implies that the radius of convergence of $\sum a_{n} z^{n}$ is at least 1 . We assume that $z$ stays inside $S_{2 \theta}$; then $t=-\log z$ satisfies

$$
|\arg (t)| \leq \frac{1}{2} \pi-\theta
$$

for small $|t|$. For $\kappa>1$ we have the integral representation [22, p. 151]

$$
\sum_{n=1}^{\infty} a_{n} e^{-n t}=\frac{1}{2 \pi i} \int_{\kappa-i \infty}^{\kappa+i \infty} D(s) \Gamma(s) t^{-s} d s
$$

Now we deform the integration contour as indicated in Figure 1, where

$$
\kappa=1-1 / \log |t|>1,
$$

and $T=T(t)>0$, to be fixed later, tends to infinity as $t \rightarrow 0$. Between $\pm i T$, the contour is defined by the function $g(\tau)$ from (9).

We will repeatedly apply the following version of Stirling's formula [10]: If $\Re(s)=\sigma$ is confined to a finite interval, then

$$
|\Gamma(s)| \sim \sqrt{2 \pi} e^{-\pi|\tau| / 2}|\tau|^{\sigma-1 / 2}, \quad|\tau| \rightarrow \infty,
$$

uniformly with respect to $\sigma$. 


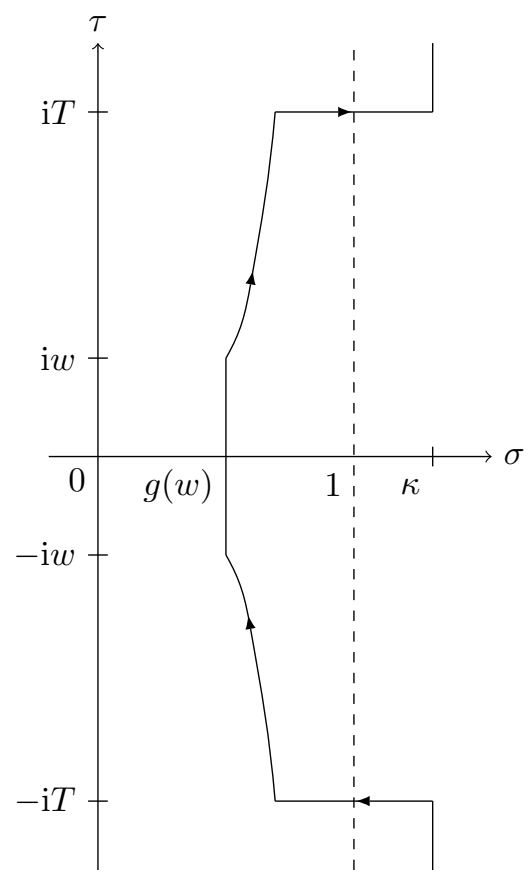

Fig. 1. The deformed integration contour, extending into the strip $0<\Re(s)<1$

To bound the integral over the upper vertical line $[\kappa+i T, \kappa+i \infty[$, note that there we have

$$
\left|\Gamma(s) t^{-s}\right| \ll_{t}|t|^{-\kappa} \tau^{\kappa-1 / 2} e^{-\tau(\pi / 2-\arg (t))} \leq e|t|^{-1} \tau^{\kappa-1 / 2} e^{-\theta \tau} .
$$

(Here and in the following, we write $A \ll_{t} B$ for $A=\mathrm{O}(B)$ as $t \rightarrow 0$, where the estimate holds uniformly in $\tau$ if $\tau=\Im(s)$ is a free variable in the right-hand side $B$.) Hence the integral over the upper vertical line satisfies

$$
\left|I_{\text {vert }}\right| \ll_{t} \frac{1}{|t|} \int_{T}^{\infty} e^{-\theta \tau} \tau^{\kappa+\nu-1 / 2} d \tau \ll_{t}|t|^{-1} e^{-\theta T} T^{\kappa+\nu-1 / 2} .
$$

We next estimate the contribution of the horizontal segment $[g(T)+i T$, $\kappa+i T$ [ to the integral. In this range we have

$$
\left|t^{-s}\right| \leq|t|^{-\kappa} e^{T(\pi / 2-\theta)}=|t|^{-1} e^{T(\pi / 2-\theta)+1}
$$

and

$$
|\Gamma(s)| \ll_{t} T^{\kappa-1 / 2} e^{-T \pi / 2},
$$

hence this portion of the integral is

$$
\left|I_{\text {hor }}\right| \ll_{t}|t|^{-1} e^{-\theta T} T^{\kappa+\nu-1 / 2},
$$

so that we obtain the same estimate as in (11). 
Finally, we bound the integral over the arc $\sigma=g(\tau)$, which we call $I_{\text {arc }}$. The integral from $g(w)$ to $g(w)+i w$ is plainly $\mathrm{O}\left(t^{\delta-1}\right)$ for some positive $\delta$, hence negligible compared to (12). In the remaining range $\tau>w$, we have

$$
\left|t^{-s}\right|=|t|^{-g(\tau)} e^{\tau \arg (t)} \leq|t|^{-g(T)} e^{\tau(\pi / 2-\theta)}
$$

and

$$
|\Gamma(s)| \ll_{t} \tau^{g(\tau)-1 / 2} e^{-\tau \pi / 2} \leq \tau^{g(T)-1 / 2} e^{-\tau \pi / 2},
$$

so that we have the bound

$$
\begin{aligned}
\left|I_{\text {arc }}\right| & \ll_{t}|t|^{-g(T)} T^{\nu} \int_{w}^{T} e^{-\tau \theta} \tau^{g(T)-1 / 2} d \tau \ll_{t}|t|^{-g(T)} T^{\nu} \Gamma(g(T)+1 / 2) \\
& \ll_{t}|t|^{-g(T)} T^{\nu} .
\end{aligned}
$$

To complete the proof, we have to pick $T$ wisely in order to balance the estimates 12 and $(13)$. We would like to have $T$ as large as possible in 12 , whereas (13) calls for a small $T$. We therefore choose

$$
T=\frac{\log 1 /|t|}{(\log \log 1 /|t|)^{\alpha}},
$$

which makes 12 and (13) approximately equal. The former then implies

$$
\left|I_{\text {hor }}\right| \ll_{t} \frac{1}{t} \exp \left(-\frac{(\theta-\varepsilon) \log 1 / t}{(\log \log 1 / t)^{\alpha}}\right),
$$

whereas (13) yields

$$
\left|I_{\text {arc }}\right| \ll_{t} \frac{1}{t} \exp \left(-\frac{(b-\varepsilon) \log 1 / t}{(\log \log 1 / t)^{\alpha}(\log \log \log 1 / t)^{\beta}}\right),
$$

both for arbitrarily small $\varepsilon>0$.

4. Further examples. Besides (5), Theorem 4 yields also estimates for other number-theoretic power series. In what follows, we let $\Lambda, \lambda, \omega$, and $\tau$ denote, as usual, the von Mangoldt function, the Liouville function, the number-of-distinct-prime-factors function, and the number-of-divisors function. Applying Theorem 4 to the Dirichlet generating functions [11, 21]

$$
\begin{aligned}
\sum_{n=1}^{\infty} \frac{(-1)^{n+1} \mu(n)}{n^{s}} & =\frac{1}{\zeta(s)} \frac{2^{s}+1}{2^{s}-1}, \\
\sum_{n=1}^{\infty} \frac{\Lambda(n)-1}{n^{s}} & =-\frac{\zeta^{\prime}(s)}{\zeta(s)}-\zeta(s), \\
\sum_{n=1}^{\infty} \frac{\lambda(n)}{n^{s}} & =\frac{\zeta(2 s)}{\zeta(s)}
\end{aligned}
$$




$$
\begin{aligned}
& \sum_{n=1}^{\infty} \frac{(-1)^{n+1} \lambda(n)}{n^{s}}=\left(1+2^{1-s}\right) \frac{\zeta(2 s)}{\zeta(s)} \\
& \sum_{n=1}^{\infty} \frac{2^{\omega(n)}-\tau(n)}{n^{s}}=\frac{\zeta(s)^{2}}{\zeta(2 s)}-\zeta(s)^{2}
\end{aligned}
$$

yields the following result.

Corollary 5. Let $E(z)$ denote the function in the error term in (5). Then

$$
\begin{aligned}
\sum_{n=1}^{\infty}(-1)^{n} \mu(n) z^{n} & =\mathrm{O}(E(z)) \\
\sum_{n=1}^{\infty} \Lambda(n) z^{n} & =\frac{1}{1-z}+\mathrm{O}(E(z)) \\
\sum_{n=1}^{\infty} \lambda(n) z^{n} & =\mathrm{O}(E(z)) \\
\sum_{n=1}^{\infty}(-1)^{n} \lambda(n) z^{n} & =\mathrm{O}(E(z)), \\
\sum_{n=1}^{\infty} 2^{\omega(n)} z^{n} & =\frac{1}{1-z} \log \frac{1}{1-z}+\frac{1+\gamma}{1-z}+\mathrm{O}(E(z)),
\end{aligned}
$$

as z tends to 1 in an arbitrary sector of the form (3).

Proof. The Dirichlet series (14)-(18) satisfy the assumptions of Theorem 4 s see, e.g., Titchmarsh [22]. As for the case of $2^{\omega(n)}$, formula (1) provides the required expansion of $\sum \tau(n) z^{n}$.

Recall that Selberg and Delange [21, II.5] established expansions for summatory functions $\sum_{n \leq x} a_{n}$ in the scale

$$
x(\log x)^{\rho-k}, \quad k=1,2, \ldots,
$$

assuming that the corresponding Dirichlet series $\sum a_{n} n^{-s}$ is sufficiently close to a power $\zeta(s)^{-\rho}$ of the zeta-function, where $\rho \in \mathbb{C}$. This is proved from Perron's summation formula, using a contour akin to Figure 1, but circumventing the possible singularity at $s=1$ by a narrow loop. The same programme could be carried out for power series, too, but this seems not worthwhile. Note that Dirichlet series with a pole at $s=1$ can be handled by Theorem 4 after subtracting a singular element, as we did in the proof of (19). An algebraic singularity at $s=1$ leads to an infinite expansion of the summatory function in the scale (20), which readily translates into an expansion for $\sum a_{n} z^{n}$ at $z=1$ by an Abelian theorem (Lemma 1). 
5. Open problems. As noted in the introduction, the unit circle is a natural boundary of $\sum \mu(n) z^{n}$. Hence one would expect that, if $z$ tends to 1 along a path that comes very close to the unit circle, the function picks up too much growth from neighboring singularities to be bounded in any scale involving only $1 /(1-z)$. So the restriction of $z$ to sectors in Theorem 4 is presumably essential. More precisely, we pose the following question: If $f: \mathbb{R}^{+} \rightarrow \mathbb{R}^{+}$is an arbitrary function, does it follow that

$$
\sum_{n=1}^{\infty} \mu(n) z^{n}=\Omega\left(f\left(\frac{1}{1-z}\right)\right)
$$

as $z \rightarrow 1$ in the unit disk?

On another register, a natural continuation of the transcendence results mentioned in the introduction would be to investigate whether the power series $\sum f(n) z^{n}$, with $f$ any of the classical arithmetic functions, can satisfy an algebraic differential equation [17].

Acknowledgements. I thank Philippe Flajolet and Florian Luca for helpful comments.

This work was financially supported by CDG, BA-CA, AFFA, and the joint centre Microsoft Research-INRIA.

\section{References}

[1] T. M. Apostol, Introduction to Analytic Number Theory, Springer, 1976.

[2] W. D. Banks, F. Luca, and I. E. Shparlinski, Irrationality of power series for various number theoretic functions, Manuscripta Math. 117 (2005), 183-197.

[3] P. T. Bateman and H. G. Diamond, On the oscillation theorems of Pringsheim and Landau, in: Number Theory, R. P. Bambah et al. (eds.), Trends Math., Birkhäuser, 2000, 43-54.

[4] J. P. Bell and M. Coons, Transcendence of generating functions whose coefficients are multiplicative, preprint, Mar. 2009; arXiv:0903.5240v1 [math.NT].

[5] J. P. Bell, S. Gerhold, M. Klazar, and F. Luca, Non-holonomicity of sequences defined via elementary functions, Ann. Comb. 12 (2008), 1-16.

[6] B. C. Berndt and R. J. Evans, Extensions of asymptotic expansions from Chapter 15 of Ramanujan's second notebook, J. Reine Angew. Math. 361 (1985), 118-134.

[7] N. H. Bingham, C. M. Goldie, and J. L. Teugels, Regular Variation, Encyclopedia Math. Appl. 27, Cambridge Univ. Press, 1989.

[8] P. Borwein and M. Coons, Transcendence of power series for some number theoretic functions, Proc. Amer. Math. Soc. 137 (2009), 1303-1305.

[9] E. R. Canfield, C. D. Savage, and H. S. Wilf, Regularly spaced subsums of integer partitions, Acta Arith. 115 (2004), 205-216.

[10] E. T. Copson, An Introduction to the Theory of Functions of a Complex Variable, Clarendon Press, 1935.

[11] H. Delange, Sur certaines séries entières particulières, Acta Arith. 92 (2000), 59-70.

[12] P. Flajolet, S. Gerhold, and B. Salvy, On the non-holonomic character of logarithms, powers, and the nth prime function, Electron. J. Combin. 11 (2005), 1-16. 
[13] P. Flajolet, X. Gourdon, and P. Dumas, Mellin transforms and asymptotics: Harmonic sums, Theoret. Comput. Sci. 144 (1995), 3-58.

[14] K. Ford, Zero-free regions for the Riemann zeta function, in: Number Theory for the Millennium II (Urbana-Champaign, IL, 2000), A. K. Peters, Natick, MA, 2002, $25-56$.

[15] G. H. Hardy and E. M. Wright, An Introduction to the Theory of Numbers, 5th ed., Oxford Univ. Press, 1979.

[16] K. Knopp, Über Lambertsche Reihen, J. Reine Angew. Math. 142 (1913), 283-315.

[17] L. A. Rubel, A survey of transcendentally transcendental functions, Amer. Math. Monthly 96 (1989), 777-788.

[18] O. Schlömilch, Compendium der höheren Analysis, Band 2, 2nd ed., Vieweg, 1874.

[19] A. M. Sedletskiǔ, Complex variants of Abelian theorem for Laplace transform, Integral Transforms Spec. Funct. 3 (1995), 107-112.

[20] R. P. Stanley, Differentiably finite power series, Eur. J. Combin. 1 (1980), 175-188.

[21] G. Tenenbaum, Introduction to Analytic and Probabilistic Number Theory, Cambridge Stud. Adv. Math. 46, Cambridge Univ. Press, 1995.

[22] E. C. Titchmarsh, The Theory of the Riemann Zeta-Function, 2nd ed., Oxford Sci. Publ., Oxford Univ. Press; edited and with a preface by D. R. Heath-Brown, 1986.

[23] A. Walfisz, Weylsche Exponentialsummen in der neueren Zahlentheorie, Deutscher Verlag Wiss., Berlin, 1963.

[24] S. Wigert, Sur la série de Lambert et son application à la théorie des nombres, Acta Math. 41 (1917), 197-218.

Stefan Gerhold

Financial and Actuarial Mathematics

Vienna University of Technology

Wiedner Hauptstraße 8/105-1

A-1040 Vienna, Austria

E-mail: sgerhold@fam.tuwien.ac.at

and

Microsoft Research-INRIA

Orsay, France 